\title{
Proyecto colaborativo para la educación geográfica sustentable: Región de Coquimbo, Chile y Estado de Iowa, Estados Unidos
}

\author{
Fabián Araya Palacios* \\ Ximena Cortés Quezada** \\ Alex Oberle*** \\ Mollie Ullestad $^{* * * *}$
}

Recibido el 13 de julio de 2014; aceptado el 24 de febrero de 2015

\begin{abstract}
This article appreciated the link between sustainability and geography by developing a standards-based lesson plans that focus on some aspect of sustainability. Is that it is a bi-national effort between Chile and the US, thus recognizing the key international importance of sustainability and the need to connect local issues, events, and problems with the larger global context and with similar occurrences elsewhere in the world. This collaborative project engages scholars in geography education and pre-service social science/geography students at two universities, specifically the state of Iowa in the US and the region of Coquimbo in Chile.

Key words: Geography Education, sustainability, collaborative project, lesson plans.
\end{abstract}

\section{Resumo}

Neste artigo analisa-se o vínculo entre a sustentabilidade e a Geografia, por meio do desenvolvimento de planos de aulas, com base em normas que se centram em algum aspecto da sustentabilidade. É um esforço binacional entre Chile e os Estados Unidos da América reconhecendo, assim, a importância internacional da sustentabilidade e a necessidade de articular as questões locais, eventos e problemas com o contexto global e com ocorrências similares em outros lugares do mundo. Este projeto de colaboração envolve acadêmicos de Educação Geográfica e estudantes de graduação em

* Universidad de La Serena, Chile, correo electrónico: faraya@userena.cl

** Universidad de La Serena, Chile, correo electrónico: xcortesq@userena.cl

*** University of Northern Iowa, Estados Unidos, correo electrónico: alex.oberle@uni.edu

****University of Northern Iowa, Estados Unidos, correo electrónico: ullestam@uni.edu 
Ciências Sociais e Geografia em duas universidades, especificamente no estado de Iowa, nos EUA e na região de Coquimbo, no Chile.

Palavras-chave: educação geográfica, sustentabilidade, projeto colaborativo, plano de classe.

\section{Resumen}

En este artículo se aprecia el vínculo entre la sustentabilidad y la geografía a través del desarrollo de planes de clases, basados en estándares que se centran en algún aspecto de la sustentabilidad. Es un esfuerzo binacional entre Chile y Estados Unidos, reconociendo así la importancia internacional, clave de la sustentabilidad y la necesidad de conectar los temas locales, eventos y problemas con el contexto global y con ocurrencias similares en otras partes del mundo. Este proyecto de colaboración involucra a académicos de educación geográfica y estudiantes de pregrado de ciencias sociales y geografía en dos universidades, específicamente en el Estado de Iowa en Estados Unidos y en la Región de Coquimbo en Chile.

Palabras clave: educación geográfica, sustentabilidad, proyecto colaborativo, plan de clases.

\section{Introducción}

La educación geográfica debe propiciar que la relación ser humano-medio ambiente se desarrolle sobre la base de una perspectiva sustentable del espacio geográfico (Stoltman, 2004; Durán, 2004; Lidstone, 2006; Commission of Geographical Education, 2007; Araya, 2010; Armstrong, 2011; Gallagher, 2012). Para lograr este propósito, se requieren profundos cambios de estilos de vida y mayores conocimientos que promuevan la conciencia pública ambiental, la participación ciudadana y el desarrollo de habilidades cognitivas para tomar decisiones en temas relacionados con el medio ambiente y su conservación.

De acuerdo a la literatura especializada, la educación geográfica no ha logrado desarrollar cabalmente en los estudiantes habilidades cognitivas que les permita entender sistémicamente el espacio geográfico y comprender las relaciones sociedadnaturaleza desde el punto de vista del desarrollo sustentable (Herremans, 2002; Reinfried, 2011; Souto, 2012; Kwangtaek, 2013). Las habilidades cognitivas corresponden a un conjunto de operaciones mentales cuyo objetivo es que los alumnos y alumnas integren la información adquirida, básicamente a través de los sentidos, en una estructura de conocimiento que tenga pertinencia para ellos (Amestoy, 2002). Actualmente, en el contexto cultural anglosajón, el concepto que se ha desarrollado para definir y profundizar esta habilidad cognitiva se denomina Spatial Thinking. Ello implica investigar sobre las diversas modalidades y estrategias para desarrollar el 
pensamiento espacial, desde la perspectiva neurolingüística, didáctica y curricular (Gersmehl, 2005; Lee, 2012; Shin-Cooper, 2012; Stuart, 2013).

Ante esta problemática, el Programa de Educación Geográfica de la Universidad de La Serena (ULS) desarrolló durante el año 2013 el proyecto titulado "Elaboración y difusión de recursos didácticos digitales bilingües, para la enseñanza-aprendizaje de la Geografía sustentable: la Región de Coquimbo (Chile) y el Estado de Iowa (Estados Unidos)". El trabajo demostró el vínculo entre la sustentabilidad y la geografía a través del desarrollo de planificaciones de clases, basadas en estándares centradas en diversos aspectos de la sustentabilidad. El presente artículo tiene como propósito sintetizar los principales resultados del proyecto, ejemplificar la metodología aplicada y los recursos didácticos obtenidos por medio de la temática de las energías renovables estudiadas en la Región de Coquimbo y en el Estado de Iowa.

\section{Antecedentes}

El proyecto, involucró a académicos de educación geográfica y a estudiantes de pregrado de ciencias sociales y geografía en dos universidades, específicamente en el Estado de Iowa, Estados Unidos y en la Región de Coquimbo, Chile.

Los objetivos del proyecto fueron los siguientes:

Objetivo general: diseñar y elaborar materiales curriculares bilingües para el trabajo de aula, basados en los Estándares y Objetivos Fundamentales de la Enseñanza de la Geografía de Estados Unidos y Chile, desde la perspectiva del desarrollo sustentable.

\section{Objetivos especificos:}

- Diseñar planificaciones de aula y recursos didácticos en formato impreso y digital utilizando herramientas de las TICs (WebQuest), para la enseñanza y aprendizaje de la Geografía de Chile, útiles para docentes chilenos, educadores estadounidenses y de otros lugares del mundo.

- Vincular al futuro docente de Historia, Geografía y Ciencias Sociales, con el medio local, regional y global-mundial, a través de la investigación de problemas geográficos cercanos desde una perspectiva sustentable del entorno.

- Integrar equipos de trabajo, estimulando el interés por la investigación de problemas geográficos locales que tienen una expresión global, desarrollando la creatividad, la capacidad de resolución de problemas, el trabajo metódico, interés y compromiso con la realidad circundante.

El proyecto intentó desarrollar en los estudiantes habilidades y destrezas para la comprensión sistémica de problemas geográficos fundamentales para el desarrollo sustentable de la Región de Coquimbo, factibles de comparar con procesos geográfi- 
cos a nivel nacional e internacional, específicamente con el Estado de Iowa en Estados Unidos. Esta experiencia de trabajar con una perspectiva internacional, permitió una ampliación de los horizontes culturales y lingüísticos de los estudiantes, lo cual redundó en una mejor preparación para la vida profesional en el mundo globalizado en el cual nos encontramos insertos en la actualidad.

El proyecto vinculó a los estudiantes con el medio local y global, a través de la investigación, comprensión y análisis de problemas geográficos en cuatro dimensiones de la sustentabilidad, cada una de las cuales contenía una expresión temática y espacial concreta en la Región de Coquimbo. Las dimensiones y las temáticas consideradas fueron las siguientes:

Dimensión económica: se investigaron los desafíos energéticos de la Región de Coquimbo, específicamente el uso de la energía eólica; la industrialización y tecnificación de la industria pisquera en el Valle de Elqui.

Dimensión medioambiental: se focalizó en dos problemáticas, riesgos naturales asociados al riesgo de tsunami en el sector de barrio Baquedano en Coquimbo y el problema de contaminación en la bahía de La Herradura.

Dimensión social: abordó el problema de la pobreza en el área rural, centrándose en la localidad de La Higuera; y el proceso de urbanización, centrado en el crecimiento de la conurbación La Serena-Coquimbo.

Dimensión cultural: esta dimensión integró, en primer lugar, el proceso de inmigración y sus efectos espaciales; específicamente en las transformaciones del paisaje asociadas a la inmigración italiana del siglo Xx y su emplazamiento en el borde costero de las ciudades de La Serena-Coquimbo. En segundo lugar se investigaron las prácticas agrícolas locales, a través de experiencias de buenas prácticas en el uso del agua en zonas rurales de la Región de Coquimbo.

Estas mismas dimensiones fueron analizadas por un equipo de trabajo de la Universidad de Northern Iowa (UNI), luego, se realizó un estudio comparativo de ambas realidades geográficas; se elaboraron los recursos didácticos y se hicieron las respectivas traducciones (inglés o español), con el fin de que las investigaciones se incorporaran a la página web de Geographic Alliance of Iowa (GAI), dependiente de la National Geographic Society (NGS) (véase $<$ http://www.uni.edu/gai/>).

El proyecto también pretendió desarrollar habilidades y destrezas en el uso de métodos y técnicas disciplinarias geográficas y herramientas de TICs, que permitieran al estudiante, futuro profesional docente, diseñar y elaborar materiales curriculares, tales como planificaciones de aula y recursos didácticos digitales orientados a la enseñanza y aprendizajes de la geografía desde la perspectiva del desarrollo sustentable. 


\section{Temáticas geográficas regionales para la sustentabilidad}

No sólo la sustentabilidad es un tema crítico, sino también encarna dos aspectos clásicos y fundamentales de la geografía: la interacción sociedad-naturaleza y el valor de una perspectiva espacial en la solución de problemas del mundo real. Con recursos escasos, creciente presión de aumento de la población y el consumo, y las nuevas tecnologías "verdes", el concepto de sustentabilidad tiene un auge importante en la última década y abarca una amplia gama de temas y aplicaciones.

Según la Agencia de Protección Ambiental de Estados Unidos

todo lo que necesitamos para nuestra supervivencia y bienestar depende, directa o indirectamente, del medio ambiente natural. La sustentabilidad crea y mantiene las condiciones en que los seres humanos y la naturaleza podría existir en armonía productiva, que permitan el cumplimiento de los requisitos sociales, económicos y de otra índole de las generaciones presentes y futuras (EPA, 2014).

En las Normas Nacionales de Geografía recientemente actualizadas en Estados Unidos, existen temas de sustentabilidad en al menos cuatro normas. Específicamente en la Norma 16 en la cual se plantean "los cambios que ocurren en el significado, uso, distribución e importancia de los recursos" (Estándares Nacionales de Geografía, 2012). La sustentabilidad también es inherente a la Norma 18, que se centra en la interpretación de la situación presente para planificar el futuro. Las Normas 14 y 15 se refieren a la dirección de la modificación humana del medio ambiente y el impacto de los sistemas físicos en los sistemas humanos. Además de la geografía, la sustentabilidad se incluye específicamente en los Estándares de Ciencias de la próxima generación como sostenibilidad humana, que abarca aspectos tales como la disponibilidad y la gestión de los recursos naturales y el desarrollo o el perfeccionamiento de soluciones científicas para abordar estas cuestiones.

Si bien, varios aspectos de la sustentabilidad han sido adoptados por las escuelas, universidades, empresas, organizaciones y gobiernos, hay una necesidad de más material curricular basado en estándares que conecten directamente con la sustentabilidad ambiental y territorial. Por ello, el equipo del proyecto identificó ocho aspectos de la sustentabilidad que son comunes tanto para el Estado de Iowa y la Región de Coquimbo, además de ser ampliamente transferibles a otras partes del mundo (Tabla 1).

Una vez identificados los temas, los estudiantes de pedagogía de cada universidad desarrollaron planificaciones de clases para cada uno de los ocho aspectos seleccionados. En la ULS se realizaron actividades del proyecto durante los talleres de la asignatura de Geografía Regional de Chile. El objetivo del estudio fue recopilar información sobre los trabajos de campo, interpretar, analizar y desarrollar recursos educativos (planificaciones de clases y actividades de aula diseñados para trabajar 
con los medios digitales) para analizar los problemas locales relacionados con la sustentabilidad geográfica.

Participaron en el proyecto 28 alumnos de octavo semestre, todos ellos de la carrera de Pedagogía en Historia y Geografía, de la Universidad de La Serena (Figura 1). Del mismo modo en la UNI, dos estudiantes de geografía y ciencias sociales trabajaron de forma independiente para crear lecciones de clases para los ocho temas. Siguiendo el ejemplo de sus homólogos chilenos, los estudiantes de formación docente crean tanto una WebQuest y un plan de clase. Con la WebQuest abordan la cuestión más amplia del tema y el plan de clases presentó el tema de la sustentabilidad a nivel del Estado (Iowa). A continuación se presentará una síntesis de los principales resultados del proyecto en los aspectos conceptuales, didácticos y metodológicos relacionados con la sustentabilidad ambiental regional.

\section{Tabla 1}

Themes and Focus Areas for Lesson Plans

\begin{tabular}{|c|c|c|}
\hline General Theme & $\begin{array}{l}\text { Lesson Plan focus for } \\
\text { Coquimbo, Chile }\end{array}$ & $\begin{array}{c}\text { Lesson plan focus for } \\
\text { Iowa, USA }\end{array}$ \\
\hline Economic-Energy & $\begin{array}{l}\text { Energy challenges in the } \\
\text { region }\end{array}$ & Wind energy \\
\hline Economic-Industrialization & $\begin{array}{l}\text { Growth of the Pisco } \\
\text { industry }\end{array}$ & $\begin{array}{l}\text { "Green" } \\
\text { Manufacturing }\end{array}$ \\
\hline Environmental-Hazards & $\begin{array}{l}\text { Tsunami hazards in } \\
\text { Coquimbo Bay }\end{array}$ & Flood hazards in Iowa \\
\hline Environmental-Pollution & $\begin{array}{l}\text { Pollution in La Herradura } \\
\text { Bay }\end{array}$ & $\begin{array}{l}\text { Urban runoff in Cedar } \\
\text { Falls }\end{array}$ \\
\hline Social-Poverty & $\begin{array}{l}\text { Rural Poverty in } \\
\text { La Higuera }\end{array}$ & Rural poverty in Iowa \\
\hline Social-Urbanization & $\begin{array}{l}\text { Urban growth in } \\
\text { La Serena-Coquimbo }\end{array}$ & $\begin{array}{l}\text { Des Moines urban } \\
\text { change over time }\end{array}$ \\
\hline Cultural-Immigration & $\begin{array}{l}\text { Italian immigration to the } \\
\text { region }\end{array}$ & Immigration in Iowa \\
\hline Cultural-Agricultural & $\begin{array}{l}\text { Water usage in rural } \\
\text { Coquimbo }\end{array}$ & Alternative agriculture \\
\hline
\end{tabular}

Fuente: Elaboración propia. 


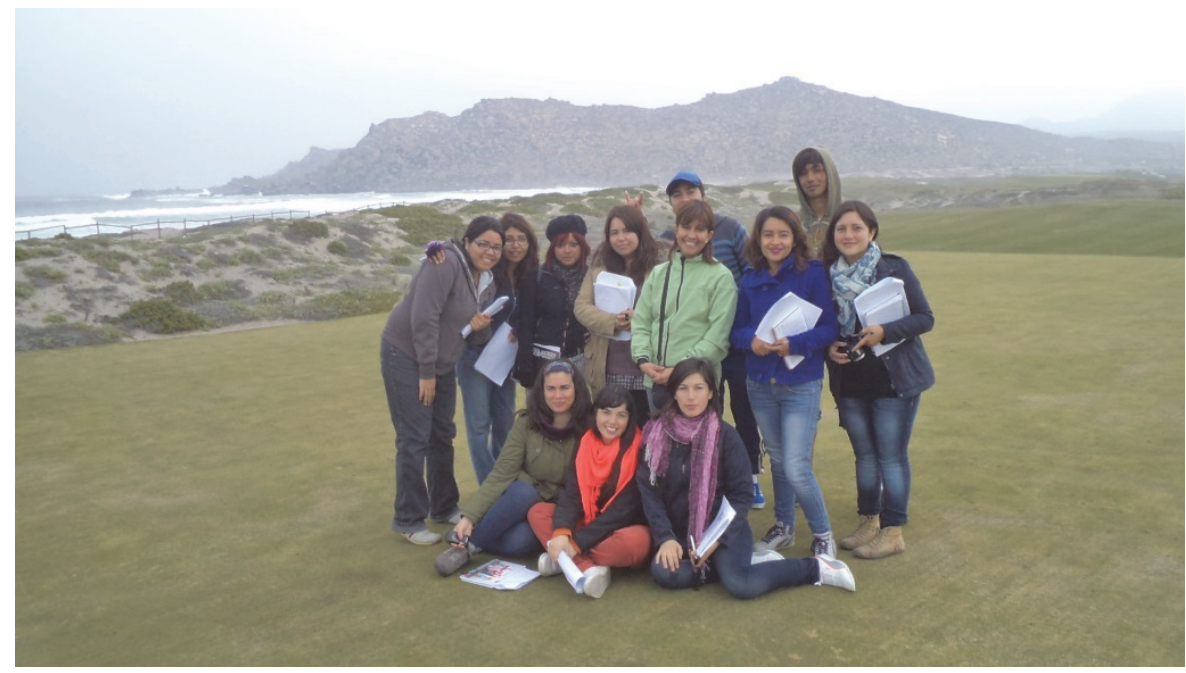

Figura 1. Estudiantes de la ULS participantes en el proyecto (Fotografía: Ximena Cortés).

\section{Resultados}

\section{Aspectos conceptuales}

Los estudiantes realizaron una investigación que les permitió conceptualizar adecuadamente los temas identificados en la matriz del proyecto (Tabla 1). Con respecto a los desafíos energéticos, la Región de Coquimbo demanda cada vez más energía eléctrica para mantener el estándar de vida que se ha construido a lo largo del tiempo. Para enfrentar esta situación, la Comuna de Canela posee un parque eólico que aprovecha las características climáticas del sector, para generar una considerable cantidad de energía eléctrica. Sin embargo, el país y la región dependen en gran parte de recursos fósiles para generar energía, por lo cual es de gran importancia educar a las nuevas generaciones respecto al tema energético y desarrollar conciencia en torno al cuidado de la energía y el medio ambiente (Jara, 2010).

Las energías renovables son energías inagotables que no dañan la biosfera al ser propias del medio natural, y que según la asociación de la Liga Municipal de Virginia es aquella suministrada a partir de fuentes de energía renovables, como la eólica y la solar, geotérmica, hidroeléctrica y de las diversas formas de biomasa. Estos principios de energía se consideran fuentes de energía renovables debido a que su origen de combustible está continuamente reponiéndose (Krumpel y Meisen, 2009).

La importancia de la utilización de energías renovables radica en la fuente limpia de energía para la preservación del espacio natural en que se vive, por lo cual requiere conciencia del riesgo e iniciativas para planificar e implementar proyectos, trayendo frutos a largo plazo y justificando la inversión. Una de las razones para implementar 
dichas energías es por su "dióxido de carbono neutral", es decir, no emiten tanto dióxido de carbono $\left(\mathrm{CO}_{2}\right)$ o más de lo que las plantas pueden asimilar en su proceso de crecimiento. Con la ayuda de tecnologías modernas, esta energía se utilizaría en forma de electricidad, calor y combustibles, donde su potencial no será agotado.

El área de estudio se centró específicamente en la Comuna de Canela correspondiente a la provincia del Choapa en la Región de Coquimbo, Chile, a $295 \mathrm{~km}$ al norte de Santiago. En esta comuna se encuentran dos principales parques eólicos, Canela I en el kilómetro 298 y Canela II al sur del primero (Figura 2).

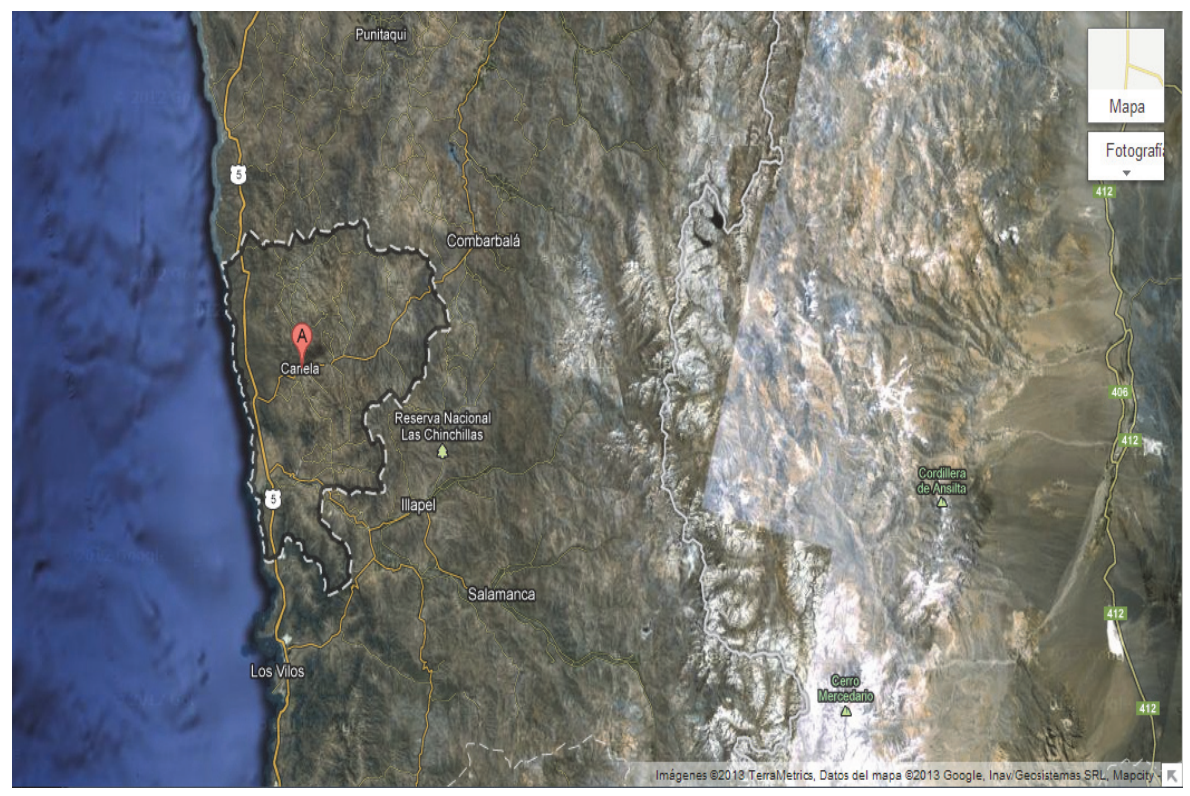

Figura 2. Localización de la Comuna de Canela.

Fuente: Google Earth, 2014.

\section{Aspectos didácticos}

En el aspecto didáctico, los estudiantes realizaron una aplicación curricular de las temáticas en diferentes programas de los cursos. Para ello se realizó una planificación de clases (Lesson Plans en el caso norteamericano). La planificación consideró el currículum vigente en cada país y las características geográficas estudiadas en la parte conceptual, por ejemplo, respecto a los desafíos energéticos de la Región de Coquimbo, la planificación se vinculó con el curso de $7^{\circ}$ año básico. A continuación se presentan, a modo de ejemplos, parte de las planificaciones o Lesson Plans desarrolladas por los estudiantes de pedagogía de ambos países (véanse Tablas 2 y 3 ). 
Tabla 2

Desafíos energéticos en la Región de Coquimbo

Curso: $7^{\circ}$ año básico, niños de 12 a 13 años

Contenido del área: energías renovables

Unidad de aprendizaje: desafíos energéticos para el mundo globalizado

Conexiones con otras disciplinas: Historia, Ciencias Naturales, Física, Economía y Política

Duración: tres días. Tres clases de 90 minutos cada una

Meta: orientar a los alumnos hacia el uso consciente de energía y la valoración de las energías limpias

Objetivos:

- Comprender los desafíos que tiene la ciudadanía para lograr un desarrollo sustentable

- Identificar el impacto y las consecuencias de las políticas públicas con respecto a la utilización de los recursos energéticos

- Valorar el uso eficiente de energías limpias para el cuidado del medio ambiente dentro de la región

Fuente: Elaboración propia.

Tabla 3

Lesson Plans: Alternative Energy, Iowa, USA

Title: The "Power" of Wind

Author: Mollie Ullestad

Affiliation: University of Northern Iowa

Grade Band

Geography for Life Standard: How to use maps and other geographic representations, tools, and technologies to acquire, process, and report information from a spatial perspective

Duration: $250 \mathrm{~min}$. class periods

Lesson Purpose and Description:

- The purpose of this lesson plan is to teach students about the process of converting wind power into electricity and where wind farmed are located in Iowa and why

- The first part of the lesson gives students the opportunity to learn about how wind turbines function and generate electricity and where wind farms are located in Iowa and why. The second part of the lesson gives students the opportunity to research their own electrical company and whether or not they use wind energy to make electricity. Students will then create a proposal. If their electrical company does not use wind power, students will propose why they should do so using data. If their company does use wind power, students will propose a new location for a wind turbine using data

- This lesson gives students the opportunity to use deductive reasoning about the relationship between, location, place, wind, and energy

- Students need to be able to read a map and be able to compare maps of the same place depicting different data. Students also need a general knowledge of geography and geographic vocabulary

Fuente: Elaboración Mollie Ullestad. 


\section{Aspectos metodológicos}

En esta sección se enfatizó el trabajo con guías de terreno y elaboración de recursos digitales, específicamente WebQuest. Por ejemplo en el caso de las energías renovables, la propuesta se concretó por medio de una guía de aprendizaje en una salida a terreno integrada, titulada "Desafíos energéticos en la Región de Coquimbo. Parque eólico de Canela”. Las principales características de la guía fueron las siguientes:

Curso: $7^{\circ}$ año (12 a 13 años de edad)

\section{Objetivos}

- Comprender los desafíos que tiene la ciudadanía para lograr un desarrollo sustentable

- Identificar el impacto y las consecuencias de las políticas públicas con respecto a la utilización de los recursos energéticos

- Valorar el uso eficiente de energías limpias para el cuidado del medio ambiente en la región

\section{Conceptos}

- Energía

- Energías renovables o limpias

- Energía eólica

- Crisis energética

- Recursos energéticos

- Desarrollo sustentable

- Efecto antrópico

\section{Habilidades}

- Localización de buenas prácticas energéticas en el ámbito local y regional

- Observación del espacio regional

- Análisis del impacto de los procesos naturales y humanos

- Formulación de opiniones y argumentos en temáticas medioambientales

- Reconocer y valorar el uso de energías limpias

\section{Desarrollo}

En funcionamiento desde diciembre de 2007, los aerogeneradores eólicos procedentes de Dinamarca, cuyo potencial es capaz de entregar energía eléctrica a 30,000 personas, ha convertido al parque eólico de Canela en el más grande del país (véase Tabla 4). 
Tabla 4

\section{Características de Canela I y II}

\begin{tabular}{ll}
\hline \multicolumn{1}{c}{ Canela I } & \multicolumn{1}{c}{ Canela II } \\
\hline Potencia: $11 \times 1.65=18.15 \mathrm{MW}$ & Potencia: $40 \times 1.5=60 \mathrm{MW}$ \\
Diámetro máx rotor: $82 \mathrm{~m}$ & Diámetro máx rotor: $82 \mathrm{~m}$ \\
Altura de torre: $70 \mathrm{~m}$ & Altura de torre: $79 \mathrm{~m}$ \\
Generación anual: $47.7 \mathrm{GWh}$ & Generación anual: $137 \mathrm{GWh}$ \\
Superficie terreno: $125.07 \mathrm{ha}$ & Superficie terreno: $1,082 \mathrm{ha}$ \\
Puerto más cercano: Coquimbo $(167 \mathrm{~km})$ & Puerto más cercano: Coquimbo $(167 \mathrm{~km})$ \\
Puesta en servicio: diciembre 2007 & Puesta en servicio: diciembre 2009 \\
\hline
\end{tabular}

Fuente: Elaboración propia.

\section{Actividades}

Responda las siguientes preguntas:

¿Qué beneficios entrega el parque eólico a la Comuna de Canela?

¿Qué impacto medio ambiental observa con la construcción del parque eólico?

¿Cuáles son los desafíos a largo plazo del proyecto del parque eólico?

\section{Entrevista}

Entreviste a un residente de la Comuna de Canela para conocer su apreciación respecto al parque eólico de Canela. Posibles preguntas:

¿Qué entiende por energía sustentable?

¿Qué beneficios considera usted que ha traído a su entorno la implementación de la energía eólica?

¿Cree usted que el parque eólico ayuda a la conservación del medio natural?

\section{Reflexión grupal}

En grupos de cuatro personas realice una breve reflexión sobre la importancia de la energía eólica para la Región de Coquimbo y elabore una propuesta concreta para fomentar y mejorar el uso de la energía eólica.

Elaborar un informe que contenga: introducción, desarrollo de la guía, evidencias de la salida a terreno, conclusiones (véase Figura 3).

En el caso de la elaboración de WebQuest, se presentan a continuación algunos ejemplos de estos recursos digitales contenidos como vínculos en las planificaciones o Lesson Plans elaboradas por los estudiantes chilenos y norteamericanos (véase Tablas 5 y 6$)$. 


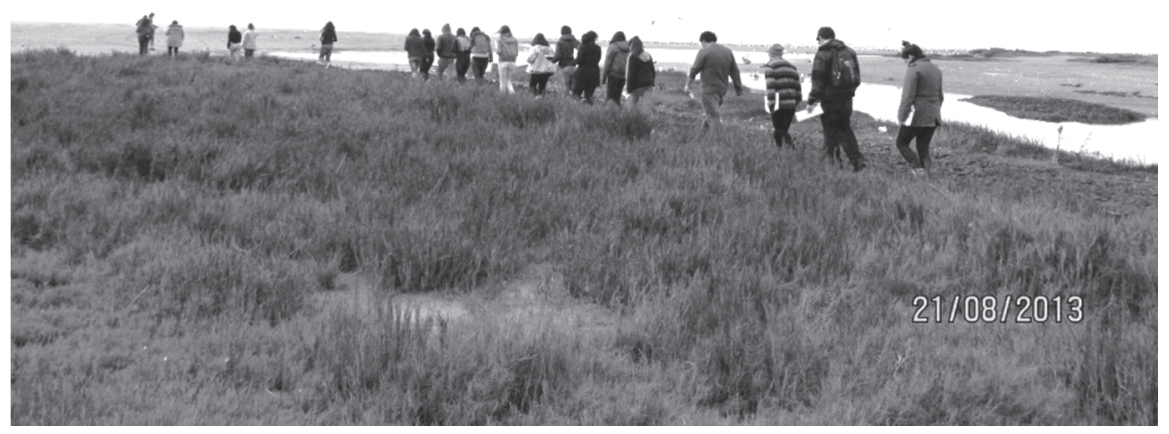

Figura 3. Estudiantes en actividad de terreno (fotografía: Ximena Cortés).

Tabla 5

Vínculos de WebQuest para desafíos energéticos en la Región de Coquimbo

Recursos y materiales requeridos:

Proyector de multimedia

Computador

WebQuest $<$ http://webquest.carm.es/majwq/wq/ver/52685>

Video didáctico $<$ http://www.youtube.com/watch? $\mathrm{v}=21 \mathrm{yhKorfO9g}>$

Cartulinas

Plumones

Prezi <http://prezi.com/_abqgi8orhkz/energias-sustentables/>

Guía didáctica de la salida a terreno

Pizarra

Planisferio

Fuente: Elaboración propia. 
Tabla 6

Vínculos de WebQuest para Alternative Energy. Iowa, USA

References and Resources

Map Sources:

$<$ http://www.broadstarwindsystems.com/state-wind-map/Iowa\%20Wind\%20Map $>$

$<$ http://geology.com/store/wall-maps/iowa.shtml $>$

$<$ http://www.earthtechling.com/2012/08/on-wind-obama-sees-a-clean-energy-winne $>$

Renewable Energy WebQuest

$<\mathrm{https}$ //sites.google.com/a/uni.edu/renewable-energy/>

Fuente: Elaboración Mollie Ullestad.

\section{Conclusiones}

El artículo ha presentado algunos de los resultados de un proyecto desarrollado entre estudiantes y profesores de la Universidad de La Serena en Chile y la Universidad de Northern Iowa en Estados Unidos, específicamente en el tema de las energías limpias y renovables. El proyecto permitió desarrollar en los estudiantes habilidades y destrezas para la comprensión sistémica de problemas geográficos fundamentales para el desarrollo sustentable de la Región de Coquimbo, factibles de comparar con procesos geográficos a nivel nacional e internacional, específicamente con el Estado de Iowa. Esta experiencia de trabajar con una perspectiva internacional, permitió una ampliación de los horizontes culturales y lingüísticos de los estudiantes, lo cual redundó en una mejor preparación para la vida profesional en el mundo globalizado en el cual nos encontramos insertos.

En el caso chileno, participaron 28 alumnos de octavo semestre de la carrera de Pedagogía en Historia y Geografía de la ULS. Del mismo modo en la UNI, dos estudiantes de geografía y ciencias sociales trabajaron de forma independiente para crear lecciones de clases para los ocho temas. El trabajo interdisciplinario desarrollado entre las cátedras de Metodología de la enseñanza de las ciencias sociales y Geografía general de Chile, permitió fortalecer el manejo conceptual, didáctico y metodológico para abordar problemas geográficos desde la perspectiva de la sustentabilidad geográfica en diferentes escalas de análisis.

La revisión y análisis de los estándares de enseñanza y aprendizaje de la geografía, desde la plataforma virtual de la Alianza Geográfica de Iowa (University of Northern Iowa) para los diferentes niveles del sistema educativo y su correlación con los Objetivos Fundamentales establecidos por el Ministerio de Educación (MINEDUC) en Chile, permitió que los alumnos pudieran identificar, comprender y analizar las nuevos conceptos y orientaciones didácticas de la geografía para la sustentabilidad. 
Los trabajos desarrollados por los estudiantes, permitieron fortalecer habilidades en el manejo de las TICs (uso de plataforma virtual, uso de herramientas digitales) generando recursos didácticos, factibles de utilizar en cualquier parte del mundo. Entre los principales recursos elaborados se encuentran los siguientes: matriz de correlación entre estándares de la enseñanza de la geografía del Estado de Iowa y objetivos fundamentales de geografía de segundo ciclo básico y enseñanza media; planificaciones de aula para diferentes niveles (según edad de los estudiantes); guías de aprendizaje en formato impreso y digital (disponibles en la web); pautas de evaluación (rúbricas, listas de cotejo); recopilación de sitios de Internet para abordar problemas geográficos relacionados con la sustentabilidad; WebQuest para abordar problemas geográficos regionales para trabajos en el aula.

En síntesis, el proyecto permitió desarrollar el espíritu crítico, cívico-ciudadano y propositivo del futuro profesional docente, a través de la generación de propuestas metodológicas y recursos didácticos, posibles de abordar en aulas de Chile y de Estados Unidos, vinculando, de esta manera, al estudiante con el entorno local y global de manera sincrónica e interrelacionada.

\section{Agradecimientos}

Los autores agradecen a los estudiantes y docentes que participaron en el proyecto, asimismo el apoyo de los Proyectos FONDECYT Regular N ${ }^{\circ} 1150214$ y DIULS N ${ }^{\circ}$ PR14412, de la Dirección de Investigación de la Universidad de La Serena.

\section{Bibliografía}

Armstrong, C.M., "Implementing education for sustainable development: The potential use of time-honored pedagogical practice from the progressive era of education", The Journal of Sustainability Education, no. 2, 2011.

Amestoy, M., "Research on the development and teaching of thinking skills", 2002. Disponible en: <http:/redie.ens.uabc.mx/vol4no1/contents-amestoy.html>, consultado 11 de julio de 2014.

Araya, F., Educación geográfica para la sustentabilidad, Monografías, La Serena, Editorial Universidad de La Serena, p. 159, 2010.

Commission of Geographical Education "Lucerne Declaration", 2007. Disponible en: <http://www.igu-cge.org/>, consultado el 12 de julio de 2014.

Durán, D., "El concepto de lugar en la enseñanza", 2004. Disponible en: <www. ecoportal.net/content/view/full/30984>, consultado el 12 de julio de 2014.

EPA, Environmental Protection Agency, Sustainability, 2014. Disponible en: <http:// www.epa.gov/sustainability/basicinfo.htm>, consultado el 12 de julio de 2014.

Gallagher, S. y Downs, R. (editors), Geography for Life: National Geography Standards. Geography Education National Implementation Proyect (GENIP), Washington: National Council for Geographic Education, pp. 117, 2012. 
Gersmehl, P., Teaching Geograph, New York, The Guilford Press, 2005, 278 pp.

Herremans, I., "Developing Awareness of the Sustainability Concept", The Journal of Environmental Education, vol. 34, no. 1, pp. 16-20, 2002.

Jara, W., "Parque Eólico Canela. Una experiencia pionera en la generación eólica en Chile", Endesa Eco, 2010. Disponible en: <http://www.expansiva.cl/media/archivos/20100819182814.pdf>, consultado el 12 de julio de 2014.

Krumpel, S. and Meisen, P., "El potencial de América Latina con referencia a la Energía Renovable”, Global Energy Network Institute, 2009, 50 pp. Disponible en: <http:/www.geni.org/globalenergy/research/renewable-energy-potential-oflatin-america/el-potencial-de-america-latina-energia-renovable.pdf $>$, consultado el 12 de julio de 2014.

Kwangtaek, S. and Stoltman, J., "Education for Sustainable Develoment Within School Geography: A proposed Model”, Journal of the Korean Geographical Society, vol. 48, no. 3, Series no. 156, 2013.

Lee, J. and Bednarz, R., "Components of Spatial Thinking: Evidence from a Spatial Thinking Ability Test”, Journal of Geography, vol. 111, no. 1, pp. 15-26, 2012.

Lidstone, J. and Williams, M., Geographical education in a changing world. Past experience, current trends and future challenges, The GeoJournal Library, vol. 85, The Netherlands, Edit. Springer, 2006, 250 pp.

National Geography Standards, Geography for Life, National Geographic Society, Washington D.C., 2012, 117 pp.

Next Generation Science Standards, Next Generation Science Standards, Achieve, Washington D.C., 2014.

Reinfried, S. and Hertig, P., "Geographical education: How human-environment-society processes work". UNESCO-EOLSS Joint Committee (Ed.), Geography, encyclopedia of life support systems (EOLSS), 2011, 20 pp. Disponible en: $<$ http://www.eolss.net/sample-chapters/c01/e6-06b-46.pdf>, consultado el 26 de junio de 2014.

Shin-Cooper, J., "Has geography education research grown in the past decade? A typology review of research in the Journal of Geography, 2000-2010", 2012, 16 pp. Disponible en: <http://geography.uoregon.edu/edge/EDGE/Projects/2012/J Shin_Cooper_2012.pdf>, consultado el 26 de junio 2014.

Souto, X., "O interesse da Investigaçao na aprendizagem e didática da geografía”, en Castellar, Sonia et. al., Didática da geografía, pp. 63-84, Edit Xama, Sao Paulo, 2012.

Stoltman, J., "Scholarship and research in Geographical and environmental education", in Eleanor Rawling (coord.) Geographical Education. Expanding horizons in a shrinking Word, SAGT Journal, vol. 33, pp 12-25, Glasgow: Geographical Communications, 2004. 
Stuart, D., et. al., The people's Guide to Spatial Thinking, Washington, National council for Geographic Education, 2013, 79 pp.

US Partnership for Education for Sustainable Development, National Education for Sustainability K-12 Student Learning Standards, US Partnership, Washington D.C., 2009. 